\title{
Metric Projections and Best Approximants in Bochner-Orlicz Spaces
}

\author{
RYSZARD PLUCIENNIK ${ }^{1}$ and YUWEN WANG ${ }^{2}$
}

\begin{abstract}
In the first section of this paper there are given criteria for strict convexity and smoothness of the Bochner-Orlicz space witb the Orlicz norm as well as the Luxemburg norm. In the second one that geometrical properties are applied to the characterization of metric projections and zero mean valued best approximants to Bochner-Orlicz spaces.
\end{abstract}

\section{INTRODUCTION.}

Problems of finding best approximants are important in approximation theory and probability theory. Best approximants in the Hilbert space $L^{2}$ are known as conditional expectations; in the space $L^{p}$ for $p>1$, as $p$-preditors [1]; in the space $L^{1}$ as conditional medians [17]; in

1) Scholar of "Ministere de Recherche et de la Technologies" of France, while on leave from Technical University at Poznan, Poland. Partially supported by Grant KBN 2.1051.91.01.

2) Supported by Chinese National Science Foundation Grant.

1991 Mathematics Subject Classification: 46E30, 41 A50, 60G 25.

Editorial Complutense. Madrid, 1994. 
an order closed sublattice of the space $L^{p}$ as $p$-means [3] and in Orlicz spaces as $\Phi$-approximants [15]. Some existence problems of best approximants in Modular spaces were considered in [7] and [8]. In this paper, we characterize the best approximant in Bochner-Orlicz spaces. Bochner-Orlicz spaces are the natural generalization of classical Orlicz spaces. Our preliminaries, Section 0, give some basic concepts and facts of the theory. Section 1 is devoted to the characterization of the strict convexity and smoothness of the Bochner-Orlicz space with the Orlicz norm as well as the Luxemburg norm. Results from Section 1 are applied to proofs of main theorems included in Section 2. In Section 2 there is described metric projection $\Pi(u \mid C)$ of any element $u \notin C$ onto convex subset $C$ of the Bochner-Orlicz space. The last theorem of this paper is a theorem on representation of zero mean valued best approximant in Bochner-Orlicz space.

\section{PRELIMINARY DEFINITIONS AND LEMMAS.}

Let $(T, \Sigma, \mu)$ be a measure space with atomless, finite measure defined on $\sigma$-algebra $\Sigma$ of subsets of $T, \mathbf{R}$ the set of real numbers, $\left(X,\|\cdot\|_{X}\right)$ a reflexive real Banach space and $\left(X^{*},\|\cdot\|_{X^{*}}\right)$ be the dual space to the space $X$. Traditionally, symbol $\left\langle x, x^{*}\right\rangle$ denotes the value of the functional $x^{*}$ at the point $x \in X$. By $\mathcal{M}(T, X)$ we denote the linear space of all $\mu$-equivalence classes of strongly measurable functions $u(\cdot): T \rightarrow X$.

A convex and even function $\Phi: \mathbf{R} \rightarrow[0, \infty)$ is called an $\mathcal{N}$-function if $\Phi(0)=0, \Phi(u)>0$ for $u \neq 0$,

$$
\lim _{u \rightarrow 0} \frac{\Phi(u)}{|u|}=0 \text { and } \lim _{u \rightarrow \infty} \frac{\Phi(u)}{|u|}=\infty
$$

For every $\mathcal{N}$-function $\Phi$ we define the complementary function $\Psi: \mathbf{R} \rightarrow[0, \infty)$ by the formula

$$
\Psi(v)=\sup _{u>0}\{u v-\Phi(u)\}
$$

for every $v \in \mathbf{R}$. The function $\Psi$ is also an $\mathcal{N}$-function (see [10]). 
We say that the $\mathcal{N}$-function $\Phi$ satisfies the $\Delta_{2}$-condition (write $\left.\Phi \in \triangle_{2}\right)$ if there exist constants $K>1, u_{0}>0$ such that

$$
\Phi(2 u) \leq K \Phi(u) \text { for } u \geq u_{0}
$$

We say that the $\mathcal{N}$-function $\Phi$ satisfies the $\nabla_{2}$-condition (write $\left.\Phi \in \nabla_{2}\right)$ if the $\mathcal{N}$-function $\Psi$ complementary to $\Phi$ satisfies the $\Delta_{2}$ condition.

Denote by small letters $\phi$ and $\psi$ the right-hand derivative of $\mathcal{N}$ functions $\Phi$ and $\Psi$, respectively.

The space

$L_{\Phi}(X)=\left\{u(\cdot) \in \mathcal{M}(T, X): \exists_{k>0} I_{\Phi}(k u)=\int_{T} \Phi\left(k\|u(t)\|_{X}\right) d t<\infty\right\}$

equipped with so called Orlicz norm

$$
\|u\|_{\Phi}=\inf _{k>0} \frac{1}{k}\left[1+I_{\Phi}(k u)\right]
$$

or with equivalent to it Luxemburg norm

$$
\|u\|_{(\Phi)}=\inf \left\{k>0: I_{\Phi}\left(k^{-1} u\right) \leq 1\right\}
$$

is said to be a Bochner-Orlicz space. Elements of the space $L_{\Phi}(X)$ will be usually denoted by $u$ instead of $u(\cdot)$ if it does not lead to misunderstanding. Further, if a Bochner-Orlicz space is equipped with the Luxemburg norm, then we will denote such space by $L_{(\Phi)}(X)$. In the case $X=\mathbf{R}$, the spaces are reduced to classical Orlicz spaces (see [11] or [16]) and they are denoted shortly by $L_{\Phi}$.

Relation between spaces $L_{\Phi}(X)$ and $L_{\Phi}$ as well as $L_{(\Phi)}(X)$ and $L_{(\Phi)}$ are expressed by the following obvious lemma

Lemma 1. $u \in L_{\Phi}(X)$ iff $\|u(\cdot)\|_{x} \in L_{\Phi}$. Furthermore,

$$
\|u\|_{\Phi}=\|\| u(\cdot)\left\|_{X}\right\|_{\Phi} \text { and }\|u\|_{(\Phi)}=\|\| u(\cdot)\|x\|_{(\Phi)}
$$


for every $u \in L_{\Phi}(X)$.

Bochner-Orlicz spaces $L_{\Psi}\left(X^{*}\right)$ and $L_{(\Psi)}\left(X^{*}\right)$ are defined analagously. The next lemma shows some connexions between $L_{\Phi}(X)$ and $L_{\Psi}\left(X^{*}\right)$.

\section{Lemma 2.}

a) The following Holder inequalities

$$
\begin{gathered}
\left|\int_{T}<u(t), v(t)>d t\right| \leq\|u\|_{\Phi}\|v\|_{(\psi)}, \\
\left|\int_{T}<u(t), v(t)>d t\right| \leq\|u\|_{(\Phi)}\|v\|_{\Psi}
\end{gathered}
$$

hold for every $u \in L_{\Phi}(X)$ and $v \in L_{\Psi}\left(X^{*}\right)$;

b) If $\Phi \in \triangle_{2}$, then $\left(L_{\Phi}(X)\right)^{*}=L_{(\Psi)}\left(X^{*}\right)$ and $\left(L_{(\Phi)}(X)\right)^{*}=$ $L_{\Psi}\left(X^{*}\right)$;

c) The space $L_{\Phi}(X)$ is reflexive iff $\Phi \in \triangle_{2} \cap \nabla_{2}$, i.e. $\Phi \in \triangle_{2}$ and $\Phi \in \nabla_{2}$.

The proof of Lemma 2 can be found in the monograph [20].

It is well known that the infimum in the definition of the Orlicz norm is realized for some $k>0$. That fact is very useful in the theory of classical Orlicz spaces. It is also true in the case of Orlicz-Bochner spaces. More precisely, there holds the following

Lemma 3. If there exists a $k_{0}>0$ such that

$$
\int_{T} \Psi\left[\phi\left(k_{0}\|u(t)\| x\right)\right] d t=1,
$$

then

$$
\|u\|_{\Phi}=\int_{T}\|u(t)\|_{X} \phi\left(k_{0}\|u(t)\|_{X}\right) d t
$$


b) For every $u \in L_{\Phi}(X) \backslash\{0\}$ there exists a $k_{0}>0$ such that

$$
\|u\|_{\Phi}=\frac{1}{k_{0}}\left\{1+\int_{T} \Phi\left(k_{0}\|u(t)\|_{x}\right) d t\right\}
$$

Proof. The proof of a) is an inmediate consequence of Theorem 1.25 from [20] and Lemma 1. By Lemma 1, b) follows from Theorem 1.27 in $[20]$.

Definition 1. (cf. [5]). Let $E$ be a Banach space, $E^{*}$ its dual space and $S\left(E^{*}\right)$ the unit sphere of $E^{*}$. A multi-valued mapping $\lambda_{E}: E \backslash\{0\} \rightarrow S\left(E^{*}\right)$ defined by the formula

$$
\lambda_{E}(u)=\left\{u^{*} \in S\left(E^{*}\right):\left\langle u, u^{*}\right\rangle=\|u\|, u \in E \backslash\{0\}\right\}
$$

is called a support mapping of $E$.

Definition 2. (cf. [2]). Let $E$ and $E^{*}$ be as in Definition 1. A multi-valued mapping $F_{E}: E \rightarrow E^{*}$ defined by the formula

$$
F_{E}(u)=\left\{u^{*} \in E^{*}:<u, u^{*}>=\|u\|^{2}=\left\|u^{*}\right\|^{2}, u \in E\right\}
$$

is called a duality mapping of $E$.

Remark 1. A relation between the support mapping of $E$ and the duality mapping of $E$ is expressed by the following formula.

$$
F_{E}(u)=\|u\| \Lambda_{E}(u) \text { for every } u \in E,
$$

where

$$
\Lambda_{E}(u)= \begin{cases}\lambda_{E}(u) & \text { for } u \in E \backslash\{0\} \\ 0^{*} & \text { for } u=0\end{cases}
$$

and $0^{*}$ is the zero element in $E^{*}$.

It turns out that the properties of $F_{E}$ are closely related to the geometry of the space $E$. The following results may be found in [2] 
Lemma 4. Let $F_{E}$ be a duality mapping of the Banach space $E$. Then

a) $F_{E}$ is surjective iff $E$ is reflexive;

b) $F_{E}$ is single-valued iff $E$ is smooth;

c) $F_{E}$ is injective iff $E$ is strictly convex.

Definition 3. Let $C$ be a convex subset of a Banach space $E$. The multi-valued mapping $\Pi(C \mid \cdot): E \rightarrow C$ defined for each $u \in E$ by the formula

$$
\Pi(C \mid u)=\left\{u_{0} \in C:\left\|u-u_{0}\right\|=\inf _{v \in C}\|u-v\|\right\}
$$

is called a metric projection onto $C$. If $\Pi(C \mid \cdot)$ is single-valued, then it is called the best approximate operator and $\Pi(C \mid u)$ the best approximant of u. In particular, if $C$ is a linear subspace of the space $E=L^{2}(T, \Sigma, \mu)$, then $\mathrm{I}(C \mid u)$ is said to be a generalized conditional expectation of $u$.

The set $\Pi(C \mid u)$ is characterized by the following lemma.

Lemma 5. (cf. [2]) Let $u_{0}$ be an element of a convex subset $C$ of a smooth Banach space $E$ and let $u \in E \backslash C$. TFAE.

a) $u_{0} \in \Pi(C \mid u)$;

b) $\left\langle u_{0}-w, \Lambda_{E}\left(u-u_{0}\right)>\geq 0\right.$ for every $w \in C$.

\section{STRICT CONVEXITY AND SMOOTHNESS OF BOCHNER-ORLICZ SPACES.}

We will begin the study of geometrical properties with the following theorem

Theorem 1. A Bochner-Orlicz space $L_{(\Phi)}(X)$ is strictly convex iff the following conditions are satisfied

a) $X$ is a strictly convex Banach space;

b) $\Phi \in \triangle_{2}$; 
c) $\Phi$ is strictly convex.

Proof. That result is a special case of the main theorem in [6].

A criterion for strict convexity of Bochner-Orlicz spaces with Orlicz norm is furnished by the following theorem

Theorem 2. A Bochner-Orlicz space $L_{\Phi}(X)$ is strictly convex iff the following conditions are satisfied

a) $X$ is a strictly convex Banach space;

b) $\Phi$ is strictly convex.

Proof of sufficiency. Denote by $S\left(L_{\Phi}(X)\right)$ the unit sphere of the space $L_{\Phi}(X)$. Suppose that a) and b) are satisfied. Let $u, v \in S\left(L_{\Phi}(X)\right)$ be such that $\|u+v\|_{\Phi}=2$. We have to proved that

$$
u(t)=v(t) \text { for a.e. } t \in T \text {. }
$$

To this end observe that $\|u(\cdot)\|_{X}$ and $\|v(\cdot)\|_{X}$ are elements of the unit sphere of the space $L_{\Phi}$. Since

$$
\|u(t)+v(t)\|_{x} \leq\|u(t)\|_{X}+\|v(t)\|_{X} \quad \text { for a.e. } t \in T,
$$

by the monotonicity of the Orlicz norm it follows that

$$
\begin{gathered}
2=\|u+v\|_{\Phi}=\|\| u(\cdot)+v(\cdot)\left\|_{X}\right\|_{\Phi} \leq \\
\leq\|\| u(\cdot)\|\|_{X}+\|v(\cdot)\|_{X}\left\|_{\Phi} \leq\right\| u\left\|_{\Phi}+\right\| v \|_{\Phi}=2 .
\end{gathered}
$$

Hence

$$
\|\| u(\cdot)\left\|_{X}+\right\| v(\cdot)\left\|_{X}\right\|_{\Phi}=2
$$


Since $\Phi$ is strictly convex, Theorem 2.4 from [20] implies that $L_{\Phi}$ is strictly convex and hence

$$
\|u(t)\|_{X}=\|v(t)\|_{X} \quad \text { for a.e. } t \in T
$$

Denote

$$
T_{0}=\{t \in T: u(t) \neq v(t)\} .
$$

Suppose that $\mu\left(T_{0}\right)>0$. Then $\|u(t)\|_{X}=\|v(t)\|_{X}>0$ for a.e. $t \in T_{0}$. Hence, by the strict convexity of the space $X$, we get

$$
\|u(t)+v(t)\|_{X}<2\|u(t)\|_{X} \quad \text { for a.e. } t \in T_{0} .
$$

Moreover, by Lemma $3 \mathrm{~b}$ ), constants $k_{1}$ and $k_{2}$ can be found such that

$$
1=\|u\|_{\Phi}=\frac{1}{k_{1}}\left\{1+\int_{T} \Phi\left(k_{1}\|u(t)\|_{X}\right) d t\right\}
$$

and

$$
\begin{gathered}
1=\|v\|_{\Phi}=\frac{1}{k_{2}}\left\{1+\int_{T} \Phi\left(k_{2}\|v(t)\|_{X}\right) d t\right\}= \\
=\frac{1}{k_{2}}\left\{1+\int_{T} \Phi\left(k_{2}\|u(t)\|_{X}\right) d t\right\} .
\end{gathered}
$$

Consequently, by the convexity of $\Phi$, we obtain

$$
\begin{aligned}
& 2=\frac{k_{1}+k_{2}}{k_{1} k_{2}}\left\{1+\frac{k_{2}}{k_{1}+k_{2}} \int_{T} \Phi\left(k_{1}\|u(t)\|_{X}\right) d t+\right. \\
& \left.+\frac{k_{1}}{k_{1}+k_{2}} \int_{T} \Phi\left(k_{2}\|u(t)\|_{X}\right) d t\right\} \geq \\
& \geq \frac{k_{1}+k_{2}}{k_{1} k_{2}}\left\{1+\int_{T} \Phi\left[\frac{k_{1} k_{2}}{k_{1}+k_{2}} 2\|u(t)\| x\right] d t\right\}> \\
& >\frac{k_{1}+k_{2}}{k_{1} k_{2}}\left\{1+\int_{T_{0}} \Phi\left[\frac{k_{1} k_{2}}{k_{1}+k_{2}}\|u(t)+v(t)\|_{X}\right] d t+\right.
\end{aligned}
$$




$$
\begin{gathered}
\left.+\int_{T \backslash T_{0}} \Phi\left[\frac{k_{1} k_{2}}{k_{1}+k_{2}}\|u(t)+v(t)\|_{X}\right] d t\right\} \geq \\
\geq \inf _{k>0} \frac{1}{k}\left\{1+\int_{T} \Phi\left(k\|u(t)+v(t)\|_{X}\right) d t\right\}=\|u+v\|_{\Phi} .
\end{gathered}
$$

This contradiction proves that $\mu\left(T_{0}\right)=0$, i.e.

$$
u(t)=v(t) \quad \text { for a.e. } t \in T
$$

as claimed.

Proof of necessity. Let $x, y \in S\left(L_{\Phi}\right)$ be such that $\|x+y\|_{\Phi}=2$. Fix $c \in S(X)$. Define

$$
u(t)=x(t) e, v(t)=y(t) e, \quad t \in T .
$$

Then $u, v \in S\left(L_{\Phi}(X)\right)$ and

$$
\|u+v\|_{\Phi}=\|x+y\|_{\Phi}=2 .
$$

By The strict convexity of the space $L_{\Phi}(X)$, we have

$$
u(t)=v(t) \quad \text { for a.e. } t \in T,
$$

and bence

$$
x(t)=y(t) \quad \text { for a.e. } t \in T .
$$

Therefore, $L_{\Phi}$ is strictly convex. Using Theorem 2.4 from [20], we conclude that $\Phi$ is strictly convex, i.e. b) is satisfied.

Now, suppose that a) is false. Then there exist $e_{1}$ and $e_{2}$ from the unit sphere $S(X)$ such that

$$
\left\|\epsilon_{1}+\epsilon_{2}\right\|_{X}=2 \text { and } \epsilon_{1} \neq \epsilon_{2} .
$$


Let $a$ be a positive real number such that $\left\|a \chi_{T}\right\|_{\Phi}=1$. Define

$$
u(t)=a e_{1} \chi_{T}(t), v(t)=a e_{2} \chi_{T}(t) \text { and } w(t)=\frac{u(t)+v(t)}{2}
$$

for every $t \in T$. Then

$$
u \neq v \text { and }\|u\|_{\Phi}=\|v\|_{\Phi}=\|w\|_{\Phi}=1,
$$

which contradicts to the strict convexity of the space $L_{\Phi}(X)$. Thus a) is satisfied. This completes the proof.

For later use, we present sufficient conditions for smoothness of the space $L_{(\Phi)}(X)$ in the following theorem

Theorem 3. If $\Phi \in \triangle_{2}, \phi$ is continuous and $X$ is smooth, then the Bochner-Orlicz space $L_{(\Phi)}(X)$ is smooth.

Proof. Since $X$ is reflexive and smooth, $X^{*}$ is strictly convex. Therefore, by the continuity of $\phi$ and Theorem 2, we conclude that $L_{\Psi}\left(X^{*}\right)$ is strictly convex. But $\left(L_{(\Phi)}(X)\right)^{*}=L_{\Psi}\left(X^{*}\right)$. Hence $L_{(\Phi)}(X)$ is smooth.

Taking into account the Bochner-Orlicz space with the Orlicz norm, we get the following

Theorem 4. If $\Phi \in \triangle_{2}, \phi$ is continuous and $X$ is smooth, then the Bochner-Orlicz space $L_{\phi}(X)$ is smooth.

Proof. Let $u$ be a non-zero element of the space $L_{\Phi}(X)$. By Lemma 3 b) there is $k>0$ such that

$$
1=\frac{1}{k}\left\{1+\int_{T} \Phi\left[\frac{k\|u(t)\|_{X}}{\|u\|_{\Phi}}\right] d t\right\}
$$

Moreover Lemma 2 states that $\left(L_{\Phi}(X)\right)^{*}=L_{(\Psi)}\left(X^{*}\right)$ because $\Phi \in \triangle_{2}$. Suppose that $v \in S\left(L_{(\Psi)}\left(X^{*}\right)\right)$ is such that

$$
\|u\|_{\Phi}=\int_{T}<u(t), v(t)>d t
$$


Note that

$$
\|u(t)\|_{X}=\left\langle u(t), \Lambda_{X}(u(t))\right\rangle \quad \text { for } t \in T \text {. }
$$

Using (1), (2) and the Young inequality, we have

$$
\begin{gathered}
1+\int_{T} \Phi\left[\frac{k<u(t), \Lambda_{X}(u(t))>}{\|u\|_{\Phi}}\right] d t=\int_{T} \frac{k u(t)}{\|u\|_{\Phi}}, v(t)>d t \leq \\
\leq \int_{T} \frac{k\|u(t)\|_{X}}{\|u\|_{\Phi}}\|v(t)\|_{X^{*}} d t \leq \\
\leq \int_{T} \Psi\left[\|v(t)\|_{X^{*}}\right] d t+\int_{T} \Phi\left[\frac{k\|u(t)\|_{X}}{\|u\|_{\Phi}}\right] d t \leq \\
\leq 1+\int_{T} \Phi\left[\frac{k<u(t), \Lambda_{X}(u(t))>}{\|u\|_{\Phi}}\right] d t .
\end{gathered}
$$

Taking into account the continuity of the function $\phi$ and conditions under which both sides of the Young inequality are equal, we get

$$
\|v(t)\|_{X^{*}}=\phi\left[\frac{k\|u(t)\|_{X}}{\|u\|_{\Phi}}\right] \quad \text { for a.e. } t \in T \text {. }
$$

Hence, by (3), we have

$$
\begin{gathered}
\int_{T}<\frac{k u(t)}{\|u\|_{\Phi}}, v(t)>d t=\int_{T} \frac{k\|u(t)\|_{X}}{\|u\|_{\Phi}}\|v(t)\|_{X^{*}} d t= \\
=\int_{T}<\frac{k u(t)}{\underline{\|u\|_{\Phi}}}, \phi\left[\frac{k\|u(t)\|_{X}}{\|u\|_{\Phi}}\right] \Lambda_{X}(u(t))>d t .
\end{gathered}
$$

Thus

$$
v(t)=\phi\left[\frac{k\|u(t)\|_{X}}{\|u\|_{\Phi}}\right] \Lambda_{X}(u(t)) \quad \text { for a.e. } t \in T,
$$

which implies that the support mapping is single-valued. This means that the space $L_{\Phi}(X)$ is smooth. 


\section{MAIN RESULTS.}

First theorem of this section gives the complete characterization of values of metric projection on convex subset $C \subset L_{\Phi}(X)$ at arbitrary point $u \in L_{\Phi}(X) \backslash C$.

Theorem 5. Let $\Phi \in \triangle_{2}, \phi$ be continuous, $X$ be smooth and $C$ be a convex subset of the space $L_{\Phi}(X)$. If $u_{0} \in C$ and $u \in L_{\Phi}(X) \backslash C$, then the following conditions are equivalent:

a) $u_{0} \in \Pi(C \mid u)$;

b) $\int_{T}<u_{0}(t)-w(t), \Lambda_{X}\left(u(t)-u_{0}(t)\right)>\phi\left[k\left\|u(t)-u_{0}(t)\right\| \|_{X}\right] d t \geq 0$ for any $w \in C$, where

$$
\int_{T} \Psi\left[\phi\left(k\left\|u(t)-u_{0}(t)\right\|_{X}\right)\right] d t=1 .
$$

Proof. By Theorem 4, the space $L_{\Phi}(X)$ is smooth. To simplify notations we denote $E=L_{\Phi}(X)$. Then, by Lemma 2 b), we obtain $E^{*}=L_{(\Psi)}\left(X^{*}\right)$.

Proof of implication. a) $\Rightarrow$ b). Let $u_{0} \in \Pi(C \mid u)$. Using Lemma 5 , we get

$$
\int_{T}<u_{0}(t)-w(t), \Lambda_{E}\left(u-u_{0}\right)(t)>d t \geq 0
$$

for every $w \in C$. Further, by Lemma $3 \mathrm{~b}$ ), there exists $k>0$ such that

$$
\begin{gathered}
\frac{1}{k}\left\{1+\int_{T} \Phi\left[k\left\|u(t)-u_{0}(t)\right\|_{X}\right] d t\right\}=\left\|u-u_{0}\right\|_{\Phi}= \\
=\int_{T}<u(t)-u_{0}(t), \Lambda_{E}\left(u-u_{0}\right)(t)>d t \leq \\
\leq \frac{1}{k} \int_{T} k\left\|u(t)-u_{0}(t)\right\|_{X}\left\|\Lambda_{E}\left(u-u_{0}\right)(t)\right\|_{X^{*}} d t \leq \\
\frac{1}{k}\left\{\int_{T} \Phi\left[k\left\|u(t)-u_{0}(t)\right\|_{X}\right] d t+\int_{T} \Psi\left[\left\|\Lambda_{E}\left(u-u_{0}\right)(t)\right\|_{X^{*}}\right] d t\right\} \leq
\end{gathered}
$$




$$
\leq \frac{1}{k}\left\{1+\int_{T} \Phi\left[k\left\|u(t)-u_{0}(t)\right\|_{X}\right] d t\right\}
$$

where $\left\|\Lambda_{E}\left(u-u_{0}\right)\right\|_{(\Psi)}=1$. Hence

$$
\int_{T} \Psi\left[\left\|\Lambda_{E}\left(u-u_{0}\right)(t)\right\|_{X^{*}}\right] d t=1
$$

and

$$
\begin{gathered}
\int_{T}\left\{\Phi\left[k\left\|u(t)-u_{0}(t)\right\|_{X}\right]+\Psi\left[\left\|\Lambda_{E}\left(u-u_{0}\right)(t)\right\|_{X^{*}}\right]-\right. \\
\left.-k\left\|u(t)-u_{0}(t)\right\| X_{X}\left\|\Lambda_{E}\left(u-u_{0}\right)(t)\right\|_{X^{*}}\right\} d t=0
\end{gathered}
$$

Therefore

$$
\begin{gathered}
\Phi\left[k\left\|u(t)-u_{0}(t)\right\|_{X}\right]+\Psi\left[\left\|\Lambda_{E}\left(u-u_{0}\right)(t)\right\|_{X^{*}}\right]= \\
=k\left\|u(t)-u_{0}(t)\right\|\left\|_{X}\right\| \Lambda_{E}\left(u-u_{0}\right)(t) \|_{X^{*}}
\end{gathered}
$$

for a.e. $t \in T$. Taking into account the continuity of $\phi$ and conditions under which both sides of the Young inequality are equal, we obtain

$$
\left\|\Lambda_{E}\left(u-u_{0}\right)(t)\right\|_{X^{*}}=\phi\left[k\left\|u(t)-u_{0}(t)\right\|_{X}\right] \text { for a.e. } t \in T \text {. }
$$

Hence ans by previous calculations, we have

$$
\begin{gathered}
\int_{T}<k\left(u(t)-u_{0}(t)\right), \Lambda_{E}\left(u-u_{0}\right)(t)>d t= \\
=\int_{T} k\left\|u(t)-u_{0}(t)\right\|_{X}\left\|\Lambda_{E}\left(u-u_{0}\right)(t)\right\|_{X} \cdot d t= \\
=\int_{T}<k\left(u(t)-u_{0}(t)\right), \phi\left[k\left\|u(t)-u_{0}(t)\right\|_{X}\right] \Lambda_{X}\left(u(t)-u_{0}(t)\right)>d t .
\end{gathered}
$$

Consequently

$$
\Lambda_{E}\left(u-u_{0}\right)(t)=\Lambda_{X}\left(u(t)-u_{0}(t)\right) \phi\left[k\left\|u(t)-u_{0}(t)\right\|_{X}\right]
$$


for a.e. $t \in T$. Moreover, by (5), we have

$$
\int_{T} \Psi\left[\phi\left(k\left\|u(t)-u_{0}(t)\right\|_{X}\right)\right] d t=\int_{T} \Psi\left[\left\|\Lambda_{E}\left(u-u_{0}\right)(t)\right\|_{X *}\right] d t=1 .
$$

Hence, using (4) and (6), we obtain b).

Proof of implication b) $\Rightarrow$ a). It follows immediately from Lemma 5 and the equality (6).

For the case of Bochner-Orlicz spaces equipped with the Luxemburg norm we can get the following theorem.

Theorem 6. Let $\Phi \in \triangle_{2}, \phi$ be continuous, $X$ be smooth and $C$ be a convex subset of the space $L_{(\Phi)}(X)$. If $u_{0} \in C$ and $u \in L_{(\Phi)}(X) \backslash C$, then the following conditions are equivalent:

a) $u_{0} \in \Pi(C \mid u)$

b) $\int_{T}<u_{0}(t)-w(t), \Lambda_{X}\left(u(t)-u_{0}(t)\right)>\phi\left[\frac{\left\|u(t)-u_{0}(t)\right\|_{X}}{\left\|u-u_{0}\right\|(\Phi)}\right] d t \geq 0$

for any $w \in C$.

Proof. By Theorem 3, the space $L_{(\Phi)}(X)$ is smooth. Denoting $E=L_{(\Phi)}(X)$, by Lemma 2 b), we obtain $E^{*}=L_{\Psi}\left(X^{*}\right)$.

Proof of implication a) $\Rightarrow$ b). Let $u_{0} \in \Pi(C \mid u)$. Lemma 5 implies that

$$
\int_{T}<u_{0}(t)-w(t), \Lambda_{E}\left(u-u_{0}\right)(t)>d t \geq 0
$$

for any $w \in C$. Furthermore

$$
\left\|\Lambda_{E}\left(u-u_{0}\right)\right\|_{\Psi}=1
$$


and

$$
\left\|u-u_{0}\right\|_{(\Phi)}=\int_{T}<u(t)-u_{0}(t), \Lambda_{E}\left(u-u_{0}\right)(t)>d t
$$

Using Lemma $3 \mathrm{~b}$ ), a positive number $k$ can be found such that

$$
\begin{gathered}
\frac{1}{k}\left\{1+\int_{T} \Psi\left[k\left\|\Lambda_{E}\left(u-u_{0}\right)(t)\right\|_{X^{*}}\right] d t\right\}=\left\|\Lambda_{E}\left(u-u_{0}\right)\right\|_{\Psi}=1= \\
=\int_{T}<\frac{u(t)-u_{0}(t)}{\left\|u-u_{0}\right\|_{(\Phi)}}, \Lambda_{E}\left(u-u_{0}\right)(t)>d t \leq \\
\leq \frac{1}{k} \int_{T} \frac{\left\|u(t)-u_{0}(t)\right\|_{X}}{\|u\| u_{0} t(\Phi)} k\left(u-u_{0}\right)(t) \|_{X^{*}} d t \leq \\
\leq \frac{1}{k}\left\{\frac{\int_{T} \Phi\left[\frac{\left\|u(t)-u_{0}(t)\right\|_{X}}{\left\|u-u_{0}\right\|_{(\Phi)}}\right]}{\leq \frac{1}{k}\left\{1+\int_{T} \Psi\left[k\left\|\Lambda_{E}\left(u-u_{0}\right)(t)\right\|_{X^{*}}\right] d t\right\} .}\right.
\end{gathered}
$$

It follows from the continuity of $\phi$ that

$$
\left\|\Lambda_{E}\left(u-u_{0}\right)(t)\right\|_{X^{*}}=\frac{1}{k} \phi\left[\frac{\left\|u(t)-u_{0}(t)\right\|_{X}}{\left\|u-u_{0}\right\|_{(\Phi)}}\right] \quad \text { for a.e. } t \in T
$$

Hence, by (8), we have

$$
\begin{gathered}
\int_{T}<\frac{u(t)-u_{0}(t)}{\left\|u-u_{0}\right\|_{(\Phi)}}, \Lambda_{E}\left(u-u_{0}\right)(t)>d t= \\
=\int_{T} \frac{\left\|u(t)-u_{0}(t)\right\|_{X}}{\left\|u-u_{0}\right\|(\Phi)}\left\|\Lambda_{E}\left(u-u_{0}\right)(t)\right\|_{X} d t= \\
\left.=\int_{T}<\frac{u(t)-u_{0}(t)}{\left\|u-u_{0}\right\|_{(\Phi)}}, \frac{1}{k} \phi \frac{\left[u(t)-u_{0}(t) \|_{X}\right.}{\left\|u-u_{0}\right\|_{(\Phi)}}\right] \Lambda_{X}\left(u(t)-u_{0}(t)\right)>d t .
\end{gathered}
$$


Consequently

$$
\Lambda_{E}\left(u-u_{0}\right)(t)=\frac{1}{k} \phi\left[\frac{\left\|u(t)-u_{0}(t)\right\| X}{\left\|u-u_{0}\right\|_{(\Phi)}}\right] \Lambda_{X}\left(u(t)-u_{0}(t)\right)
$$

for a.e. $t \in T$. Combining formula (9) with (7) we complete the proof of the desire implication.

Proof of implication. b) $\Rightarrow$ a). It follows immediately from Lemma 5 and formula (9).

A subspace $L \subset L_{\Phi}(X)$ is said to be a zero mean valued subspace if

$$
L=\left\{w \in L_{\Phi}(X): \int_{T} w(t) d t=0\right\}
$$

where an integration is in the Bochner sense.

Theorem 7. If $\Phi \in \triangle_{2} \cap \nabla_{2}, \phi$ and $\psi$ are continuous and $X$ is refiexive, smooth and strictly convex, then for each $u \in L_{\Phi}(X)$ there exists a unique best approximate element $\Pi(C \mid u) \in L$. Furthermone

$$
\Pi(L \mid u)=u-\frac{\chi_{T}}{\mu(T)} \int_{T} u(t) d t \quad \text { for } u \in L_{\Phi}(X) .
$$

Proof. Using Lemma 2, Theorem 2 and Theorem 4, we conclude that $L_{\Phi}(X)$ is reflexive, strictly convex and smooth. Moreover, $L$ is a linear and closed subspace of $L_{\Phi}(X)$. Indeed, $L$ is linear in an obvious manner. For the proof of the closure of $L$, suppose that $\left\{w_{n}\right\}$ is a sequence of elements of the subspace $L$ such that

$$
\left\|w_{n}-w\right\|_{\Phi} \rightarrow 0 \text { as } n \rightarrow \infty
$$

Note that $x^{*} \chi_{T}(\cdot) \in L_{(\Psi)}\left(X^{*}\right)=\left(L_{\Phi}(X)\right)^{*}$ for any $x^{*} \in X^{*}$. Hence

$$
<\int_{T} w(t) d t, x^{*}>=\int_{T}\left\langle w(t), x^{*}>d t=\int_{T}\left\langle w(t), x^{*} \chi_{T}(t)\right\rangle d t=\right.
$$




$$
\left.=\lim _{n \rightarrow \infty} \int_{T}\left\langle w_{n}(t), x^{*} \chi_{T}(t)\right\rangle d t=\lim _{n \rightarrow \infty}<\int_{T} w_{n}(t) d t, x^{*}\right\rangle=0
$$

for each $x \in X$. Therefore

$$
\int_{T} w(t) d t=0
$$

i.e. $w \in L$.

Since $L$ is a closed linear subspace of the reflexive strictly convex space $L_{\Phi}(X)$, there exists a unique element $u_{0} \in L$ such that

$$
\left\|u-u_{0}\right\|_{\Phi}=\inf _{v \in L}\|u-v\|_{\Phi}
$$

i.e. $u_{0}=\Pi(L \mid u)$.

By Theorem 5,

$$
\int_{T}<u_{0}(t)-w(t), \Lambda_{X}\left(u(t)-u_{0}(t)\right)>\phi\left[k\left\|u(t)-u_{0}(t)\right\|_{X}\right] d t \geq 0
$$

for any $w \in L$, where

$$
\int_{T} \Psi\left[\phi\left(k\left\|u(t)-u_{0}(t)\right\|_{X}\right)\right] d t=1
$$

Since $L$ is linear, we have

$$
\int_{T}<w(t), \phi\left[k\left\|u(t)-u_{0}(t)\right\|_{X}\right] \Lambda_{X}\left(u(t)-u_{0}(t)\right)>d t=0
$$

for each $w \in L$. Hence, by the definition of the duality mapping $F_{E}: E \rightarrow E^{*}$, we obtain

$$
\begin{gathered}
F_{E}\left(u-u_{0}\right)=\left\|u-u_{0}\right\|_{\Phi} \Lambda_{E}\left(u-u_{0}\right)= \\
\left\|u-u_{0}\right\|_{\Phi} \phi\left[k\left\|u(\cdot)-u_{0}(\cdot)\right\| \|_{X}\right] \Lambda_{X}\left(u(\cdot)-u_{0}(\cdot)\right) \in L^{\perp} \subset L_{(\Psi)}\left(X^{*}\right),(10)
\end{gathered}
$$


where $E=L_{\Phi}(X)$. Denote

$$
H=\left\{x^{*} \chi_{T}(\cdot): x^{*} \in X^{*}\right\}
$$

Obiously, $H$ is a closed, linear subspace of $L_{(\Psi)}\left(X^{*}\right)$. Moreover,

$$
\int_{T}<w(t), x^{*} \chi_{T}(t)>d t=<\int_{T} w(t) d t, x^{*}>=0
$$

for any $w \in L$ and $x^{*} \in X^{*}$. This inequality implies inmediately that $L=H^{\perp}$. Therefore,

$$
L^{\perp}=H^{\perp \perp}=H \text {. }
$$

Hence, by (10), there exists $x_{0}^{*} \in X^{*}$ such that

$$
F_{E}\left(u-u_{0}\right)=x_{0}^{*} \chi T .
$$

Let $F_{E^{*}}: E^{*} \rightarrow E^{* *}=E$ be the duality mapping $E^{*}$. Since $E$ is reflexive, smooth and strictly convex, $F_{E}$ and $F_{E^{*}}$ are bijections (cf. Lemma 4) and $F_{E}^{-1}=F_{E^{*}}$. Thus from (11) it follows that

$$
u-u_{0}=F_{E}^{-1}\left(x_{0}^{*} \chi_{T}\right)=F_{E^{*}}\left(x_{0}^{*} \chi_{T}\right) .
$$

Since

$$
\begin{gathered}
\int_{T}<x_{0}^{*} \chi_{T}(T), \frac{1}{\mu(T)}\left[\Psi^{-1}\left(\frac{1}{\mu(T)}\right)\right]^{-2}\left\|x_{0}^{*}\right\|_{X^{-}} \Lambda_{X *}\left(x_{0}^{*}\right) \chi_{T}(t)>d t= \\
=\frac{1}{\mu(T)} \int_{T}\left\|x_{0}^{*}\right\|_{X^{*}}^{2}\left[\Psi^{-1}\left(\frac{1}{\mu(T)}\right)\right]^{-2} d t= \\
=\left\|x_{0}^{*}\right\|_{X^{*}}^{2}\left[\Psi^{-1}\left(\frac{1}{\mu(T)}\right)\right]^{-2}=\left\|x_{0}^{*} \chi_{T}\right\|_{(\Psi)}^{2},
\end{gathered}
$$


we deduce, by the definition of $F_{E^{*}}$, that

$$
F_{E^{*}}\left(x_{0}^{*} \chi T\right)=x_{1} \chi T
$$

where

$$
x_{1}=\left\|x_{0}^{*}\right\|_{X^{*}} \Lambda_{X} \cdot\left(x_{0}^{*}\right)\left[\Psi^{-1}\left(\frac{1}{\mu(T)}\right)\right]^{-2} \frac{1}{\mu(T)} \in X^{* *}=X
$$

Combining (12) and (13), we obtain

$$
u-\Pi(L \mid u)=x_{1} \chi_{T}
$$

Integrating this equality over $T$, we obtain

$$
\int_{T} u(t) d t=\int_{T} x_{1} \chi_{T}(t) d t=x_{1} \mu(T)
$$

and hence

$$
x_{1}=\frac{1}{\mu(T)} \int_{T} u(t) d t .
$$

Coming back to the equality (14), we get inmediately

$$
\Pi(L \mid u)=u-\frac{\chi_{T}}{\mu(T)} \int_{T} u(t) d t
$$

for any $u \in L_{\Phi}(T)$. This completes the proof.

\section{References.}

[1] Ando, T. and Amemiya, I., Almost everywhere convergence of prediction sequence in $L_{p}(1<p<\infty), Z$. Wahrscheinlichkeitstheorie verw. Geb. 4 (1965), 113-120.

[2] Barbu, V. and Precupanu, T., Convexity and optimization in Banach spaces, Sijthoff and Northoff International Publishers - Ed. Acad. Bucuresti, Romania, 1978. 
[3] Brunk, H.D., Uniform inequalities for conditional p-means given $\sigma$ lattices, Ann. Probability 3 (1975), 1025-1030.

[4] Darst, R.B., Legg, D.A. and Townsent, D.W., Prediction in Orlicz spaces, Manuscripta Math. 35 (1981), 91-103.

[5] Diestel, J., Geometry of Banach spaces, Lecture Notes in Math., vol. 485, Springer-Verlag, 1975.

[6] Hudzik, H., Strict convexity of Musielak-Orlicz space with Luxemburg's norm, Bull. Pol. Ac. Math., 29 (1981), 235-247.

[7] Kilmer. S.J., Kozlowski, W.M. and Lewicki, G., Best approximants in modular function spaces, J. Approx. Theory 63.3 (1990), 338-367.

[8] Kilmer, S.J., Kozlowski, W.M. and Lewicki, G., Sigma onder continuity and best approximation in $L_{\rho}$-spaces, Comment. Math. Univ. Carolinae 32.2 (1991), 241-250.

[9] Kozek, A., Convex integral functionals on Orlicz spaces, Comment. Math 21 (1979), 109-135.

[10] Kozek, A., Orlicz spaces of functions with values in Banach spaces, Comment. Math. 19 (1976), 259-288.

[11] Krasnosel'skij, M.A. and Rutickij, Y.A., Convex functions and Orlicz spaces, Noordhoff, Gronigen, 1961.

[12] Landers, D. and Rogge, L., Characterization of p-predictors, Proc. Amer. Math. Soc. 76 (1979), 307-309.

[13] Landers, D. and Rogge, L. A short proof for a.e. convergence of generalized conditional expectations, Proc. Amer. Math. Soc. 79 (1980), 471.473 .

[14] Landers, D. and Rogge, L., Best approximants in $L_{\Phi}$-spaces, $Z$. Wahrscheinlichkeitstheorie verw. Geb. 51 (1980), 215-237.

[15] Landers, D. and Rogge, L., A characterization of best $\Phi$-approximants, Trans. Am. Math. Soc. 267 (1981), 259-264.

[16] Musielak, J., Orlicz spaces and Modular spaces, Lecture Notes in Math., vol. 1034, Springer-Verlag 1983.

[17] Shintani, T. and Ando, T., Best approximants in $L_{1}$ space, $Z$. Wahrscheinlichkeitstheorie verw. Geb. 33 (1975), 33-39. 
[18] Singer, I., Best approximation in normed linear spaces by elements of linear subspaces, Springer, 1970.

[19] Wang, Y.W., Some approximation and control problems in Orlicz spaces, Teubner-Texte zur Math. Band 103 (1988), Function Spaces Proceedings of the International Conference Poznan, 1986, 41-45.

[20] Wu, C.X., Wang, T.F., Chen, C.T. and Wang, Y.W., Geometry of Orlicz spaces (chinese), Harbin Institute of Technology, Harbin 1986.

Departamento da Matemática Universidade da Beira Interior Rua Marqués d'Avila e Bolama 6200 Covilha

Portugal

Department of Mathematics

Harbin Normal University

Hexing Road

Harbin,

China
Recibido: 12 de marzo de 1992

Revisado: 22 de septienbre de 1993 